\title{
AIDA: Web Agents in Dental Treatment Planning
}

Ekkehard Finkeissen ${ }^{a}$, Sabine Böhret ${ }^{a}$, Irmela Stamma , Miriam Müssiga, Jochen Streicher $^{a}$, Ulrich Koke ${ }^{b}$, Claudia Helmstetter ${ }^{b}$, Astrid Wolff ${ }^{a}$, Stefan Hassfeld ${ }^{b}$, Thomas Wetter ${ }^{\mathrm{a}}$

${ }^{a}$ Institute for Medical Biometry and Informatics, University of Heidelberg, Germany.

${ }^{b}$ Dental Clinic, University of Heidelberg, Germany.

\begin{abstract}
Objective of the AIDA-project (Artificial Intelligent Dental Agents, aida.uni-hd.de) is the analysis of dental decision-making, the design of a computer-based decisionsupport, and the testing of the decision structure in the interaction with dental experts, practicing dentists, and patients. The planning of the solution alternatives is based on a top-down structure for dental decision-making aiming at a standardization of the argumentation. [1]

For prosthetic dentistry, it has been clarified how one uses individual patient findings to sketch the possible treatment alternatives. The planning module for fixed prostheses have already been integrated into a software agent [2]. Other planning modules are currently specified, implemented, and verified (i.e., other types of prostheses and restorative dentistry). A structure for dentally founded justifications for every individual decision is currently integrated into the decision modules and will be specified in cooperation with dental experts soon.
\end{abstract}

Keywords:

Dental Informatics, Agents, Knowledge Management, Ontologies, Web Services

\section{The AIDA Approach}

Dental treatment planning is highly dependent on the educational background and personal experience of the dentist and on the thoroughness with which findings are taken. Therefore, a system for decision-making in dentistry as a top-down structure was developed as part of the University of Heidelberg's AIDA project to clarify planning requirements [1].

Prior approaches for dental treatment planning were restricted to partial aspects [3-9]. In contrast, AIDA's methodology aims at a top-down partitioning of dental decision-making and enables the multidimensional complexity of dentistry - consisting of, among other things, medical, technical, economic, and legal aspects - to be organized in small, easy-tohandle planning modules.

Starting with general goals for dental treatment, patient-specific target-criteria are derived from the patient's individual findings. From this information, AIDA can derive all treatment alternatives meeting the target criteria. All treatment alternatives are presented as equivalents, which allows the practicing dentist to select the individually appropriate type 
of therapy. Here, "no prosthesis necessary" is also a valid option. Since a systematic decision-making is an abstracted representation of individual cases, it cannot include all aspects. Accordingly, it is up to the dentist to select one of AIDA's treatment suggestions and examine them in detail if desired.

Since many of the fixed prosthetic solutions in dentistry involve more than one tooth, an appraisal of their necessity should be performed as early as possible - only this allows the assessment of their effects on other teeth. After the possible prosthetic treatment alternatives have been determined, detail questions can be clarified in order to test the concrete applicability of the desired prosthetic solution (i.e., periodontal requirements).

A comparison of AIDA plannings with real-life patient records has already yielded valuable indications for extensions and adaptations related to the planning rules. ${ }^{3}$ In the current version of AIDA, specific planning aspects of restorative dentistry related to the planning of a prosthetic construction are included. From this extension, a further increase of both recall and precision is expected in AIDA's planning.

\section{The current prototype to AIDA}

AIDA's planning modules were implemented incrementally as separate software agents. This made it possible to test the each planning modules separately and assign responsibilities to different experts groups. Each of these agents can answer the following questions in terms of individual treatment planning:

- How many solutions are there?

- What are they called and described?

- How do they look like?

In the next version, the AIDA agents will be able to provide information on further questions:

- What is the rationale behind deciding for a certain solution?

- How much does the solution cost?

The AIDA interface supports the anonymous coding of the findings. The information is sent to the AIDA agent where the actual planning is carried out. In the planning system, the transferred findings are checked for integrity using plausibility rules. Only after the integrity of the transferred information has been established, AIDA can suggest plans. 


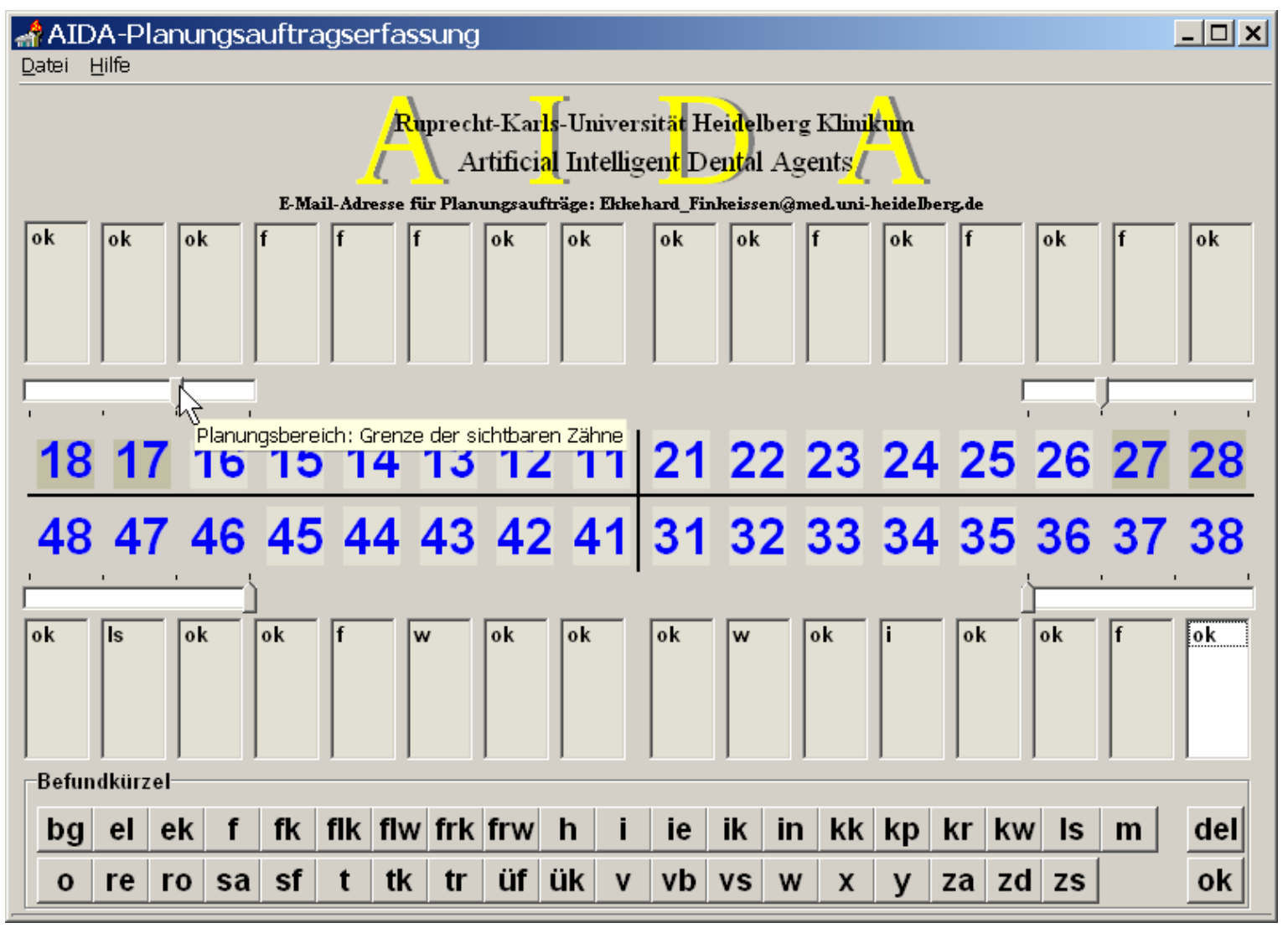

Fig. 1: the interface of AIDA is separated from the actual planning machine and can be downloaded from the Internet. A web-based version will be available soon.

The transfer of findings is currently being adapted to a new standard established within the AIDA project. After this re-implementation, the AIDA planning suggestions can be accessed both by dentists using web browsers and by other machines (e.g., dental billing software) (cp. fig 2).

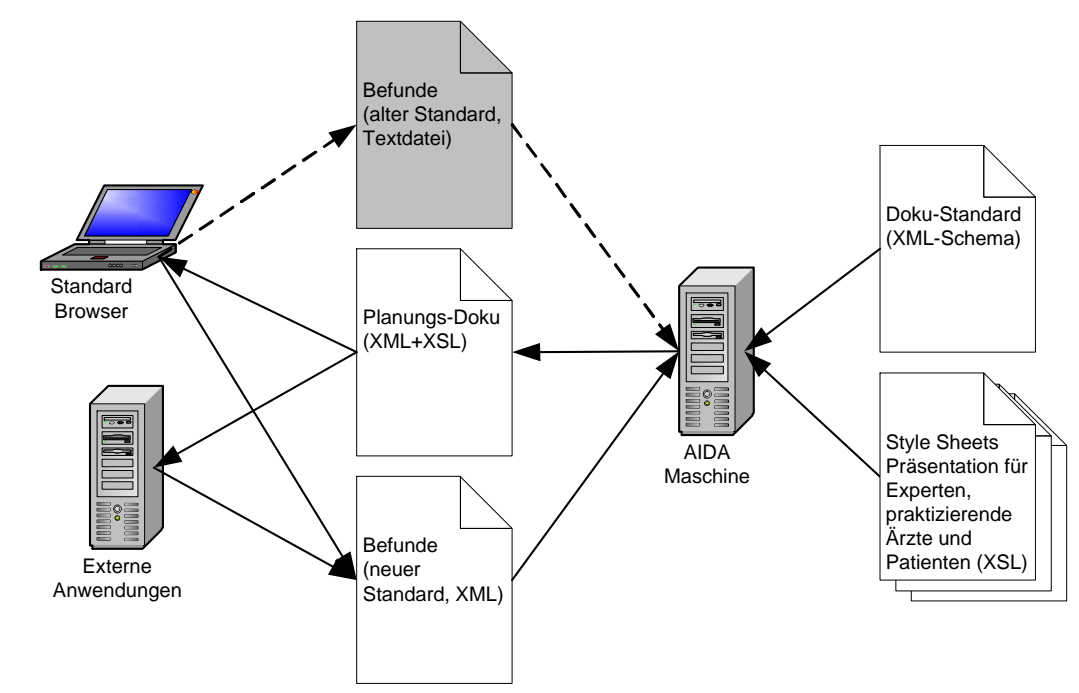

Fig 2: revised documentation abbreviations will make dentists ${ }^{6}$ communication more precise. Implemented in XML, the information can be both automatically processed and appropriately presented for the respective user group. 
In the current version, the planning suggestions are formulated in XML and can be visualized with the help of a style sheet. This allows the planning results to be adapted to the individual presentation needs to dental experts (focus: evaluation), practicing dentists (focus: planning support), or patients (focus: explanatory support). AIDA's plannings results in the output of all practicable and relevant treatment alternatives based on the given findings. These solutions can, i.e., be passed along to a billing program and shown on the planning form. The dentist can accept one of the suggestions and edit it as desired. Since the treatment options for fixed prostheses can be already be planned by AIDA based on the standard findings form, the usage does not cost the dentist any additional effort and may in many cases - even save time.

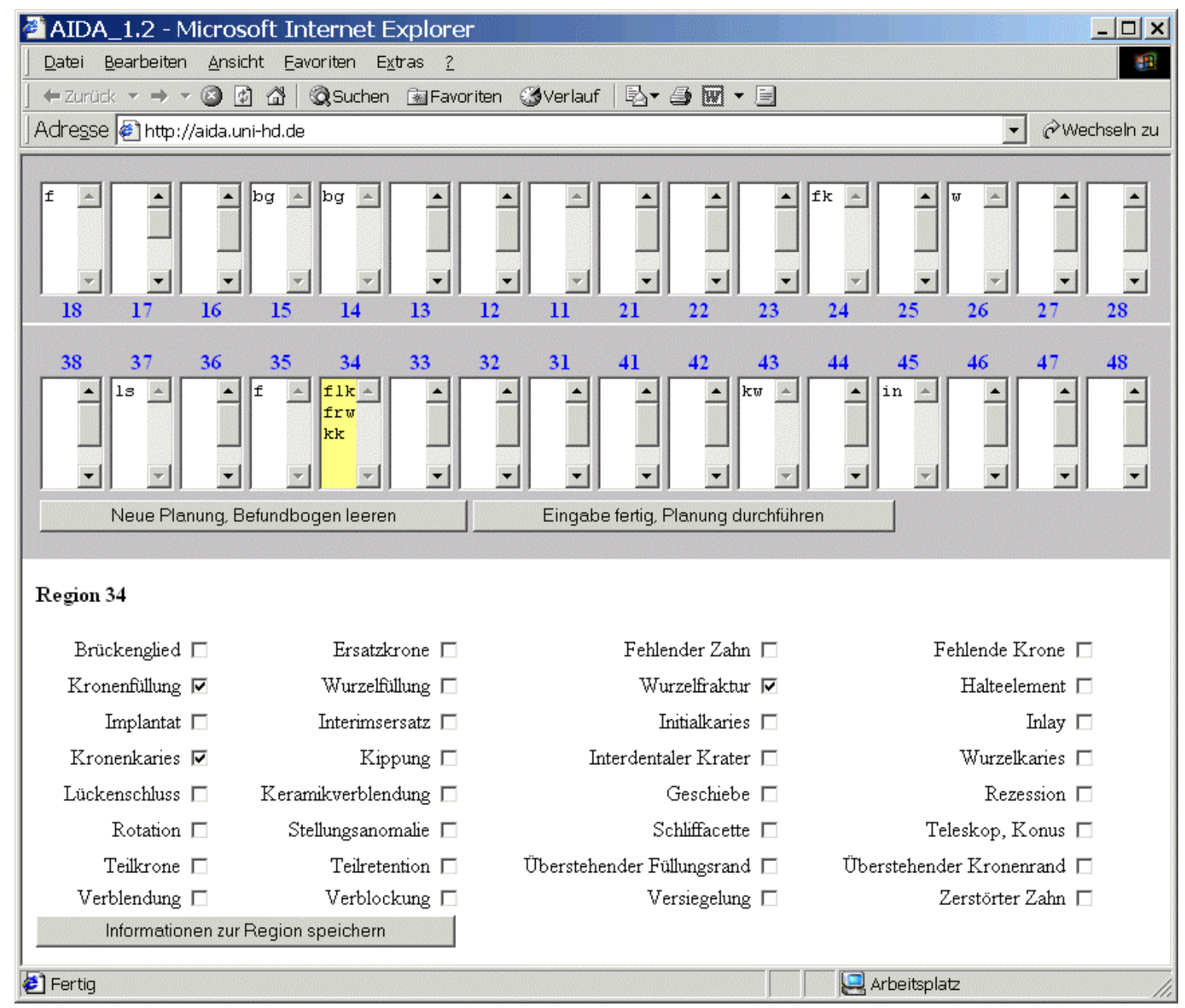

Fig 3: prototypical output of the AIDA-agent for dentists. In order to subsequently check the appropriateness of the treatment alternative, the findings and their interpretation are also included in the planning output.

Since no general rules for the evaluation of dental planning are available, a committee of dental experts was formed to serve as the golden standard for the evaluation of AIDA planning; this committee examined real cases from the patient records and approved comparison in positive cases. The first comprehensive evaluation of AIDA demonstrated that up to $68 \%$ of the AIDA suggestions were deemed practicable and relevant by dental 
experts [10]. An integration of further dental aspects is assumed to increase the planning recall (i.e., from restorative dentistry).

\section{Discussion}

The computer cannot replace the dentist. A machine cannot even be allowed to make decisions on its own, since it cannot understand the patient's problems and needs. For this reason, computer-assisted decision-making will remain a communication medium between experts and practicing doctors or dentists in the future. As a neutral authority, however, such a communication instrument can promote rational discussions between experts as well as dentist and patient. Like this, it can support explaining the necessity of complex treatment alternatives to patients.

During the development of AIDA, it became clear that a uniform and structured method of documentation for the comprehensible communication of dental decisions is essential. Fundamental deficits in the practice of dental documentation were revealed [11]. A draft for the reorganization of dental documentation is already in progress, and should be available later this year in form of a structured description in XML format.

A nation-wide expansion of the dental expert team will hopefully support the integration of a broader range of treatment alternatives into the AIDA methodology. As soon as AIDA includes a convincing number of treatment alternatives, its structure can also serve the definition of comparative studies on the evaluation of the individual treatment alternatives and they can again be included into AIDA approach later.

\section{References}

1. Finkeissen E. Zahnmedizinische Entscheidungsstrukturen - Grundlagen einer wissensbasierten Zweitmeinung: Universität Heidelberg; 2002.

2. Finkeissen E, Weber R, Hassfeld S, Koke U, Wetter T. AIDA - experiences in compensating the mutual weaknesses of knowledge- based and object-oriented development in a complex dental planning domain. Methods Inf Med 2002; 41:168-76.

3. Balch DC, Warner DJ, Gustke SS. Highlights From the Third Annual DMI Conference - Medical Knowledge on Demand. MD-Computing 1999; March/April:48-50.

4. Cordato M. A mathematical study of anterior dental relations: Part II, Incisor and canine overjet. Aust Orthod J 1996; 14(3):143-9.

5. Sellen P, Jagger D, Harrison A. Computer-generated study of the correlation between tooth, face, arch forms, and palatal contour. J Prosthet Dent 1998; 80(2):163-8.

6. Han K, Lu R, Ma Y, Lu P, Zhang H, Wang W. Reconstruction of three-dimensional digital teeth. Chin J Dent Res 1998; 1(2):22-5.

7. Pan S, Yin Y, Feng H. Three-dimensional finite element analysis and comparison of stress distribution in overdentures supported with bar attachments and telescopic crowns. Chin J Dent Res 1999; 2(1):21-30.

8. Okumura H, Chen L, Yokoe Y, Tsutsumi S, Oka M. CAD/CAM fabrication of occlusal splints for orthognathic surgery. J Clin Orthod 1999; 33(4):231-5.

9. Zhang X, Yang C, Gao J, Ding Z. Computer-assisted design and manufacture of posterior full crowns. Chin J Dent Res 1998; 1(3):30-4.

10. Finkeissen E, Koke U, Hassfeld S, Helmstetter C, Weber R, Wetter T. AIDA - Iterative Approach for the Improvement of a Knowledge-based Decision Methodology in Dentistry. British Dental Journal submitted 2002; .

11. Finkeissen E, Hassfeld S, Koke U, Helmstetter-Blasius, Eickholz P, Wetter T. Problems with Current Dental Documentation in Germany. In: . Heidelberg: Institute for Medical Biometry and Informatics, Department of Medical Informatics; 2002. p. 15. 